\title{
Management and grading of EGFR inhibitor-induced cutaneous toxicity
}

Janette Beech1, Theodora Germetaki ${ }^{1}$, Mary Judge², Nina Paton ${ }^{1}$, Joanne Collins ${ }^{1}$, Abigail

Garbutt $^{3}$, Michael Braun 1 , Jill Fenwick ${ }^{4}$ \& Mark P Saunders*,1

${ }^{1}$ Department of Medical/Clinical Oncology, Christie Hospital, 550 Wilmslow Road, Manchester, UK

${ }^{2}$ Department of Dermatology, Salford Royal NHS Foundation Trust, Salford, UK

${ }^{3}$ Pharmacy of The Christie Hospital Foundation Trust, NHS, UK

${ }^{4}$ Merck Serono Ltd., Feltham, UK (an affiliate of Merck KGaA, Darmstadt, Germany)

*Author for correspondence: Mark.Saunders@christie.nhs.uk

Cutaneous toxicities associated with EGFR inhibitors (EGFRIs) have a significant impact on patient treatment continuation, quality of life and healthcare resource utilization. This paper reviews the current prophylaxis and management of EGFRI-induced cutaneous toxicities in patients with colorectal cancer, and combines these findings with the authors' clinical expertise to define a novel algorithm for healthcare professionals managing patients receiving EGFRIs. This tool includes a grading system based on the location, severity and psychological impact, and provides a standard prescription pack, advice on prophylaxis/selfmanagement of cutaneous symptoms for patients initiating EGFRIs, and essential guidance on subsequent review and treatment escalation. It aims to optimize treatment of metastatic colorectal cancer by minimizing cutaneous toxicities to maintain dose intensity and efficacy of EGFRI-based chemotherapy.

First draft submitted: 8 March 2018; Accepted for publication: 2 April 2018; Published online: 4 May 2018

Keywords: acneiform eruptions $\bullet$ adverse reactions $\bullet$ cetuximab $\bullet$ drug-related side effects $\bullet$ EGFRI $\bullet$ metastatic colorectal cancer $\bullet$ panitumumab $\bullet$ paronychia $\bullet$ patient compliance $\bullet$ quality of life $\bullet$ receptor $\bullet$ treatment algorithm

Globally, colorectal cancer is the third most commonly diagnosed cancer [1]. Approximately a quarter of initial presentations for colorectal cancer are for metastatic disease, and $40-50 \%$ of individuals with early stage disease eventually develop metastatic colorectal cancer [2]. Metastasis contributes to the high mortality rate associated with colorectal cancer. In 2012, colorectal cancer accounted for 694,000 deaths worldwide [1], with Europe showing the highest standardized incidence and mortality rates of all six WHO regions [3].

As well as VEGF inhibitors, trifluridine/tipiracil, regorafenib and the immunotherapies, EGFR inhibitors (EGFRIs) offer a valuable treatment option for patients with metastatic colorectal cancer. The addition of EGFRIs to the combination chemotherapy agents used in metastatic disease can extend survival to around 30 months in patients with $R A S$ wild-type metastatic colorectal cancer [4,5]. EGFRIs have gained widespread acceptance as an anticancer treatment and are associated with a different spectrum of adverse events compared with those seen with chemotherapy [6]. These adverse events are usually minimal in terms of frequency and severity, with the exception of cutaneous toxicities, which perhaps present the greatest concern with EGFRI use. This article focuses on EGFRIs, their skin-associated toxicities and management strategies to maximize the treatment benefit of EGFRIs in metastatic colorectal cancer.

EGFRIs for the treatment of metastatic colorectal cancer

Two EGFRIs are currently indicated for use in metastatic colorectal cancer in Europe: cetuximab and panitumumab. European Society for Medical Oncology (ESMO) guidelines suggests their use in combination therapy at any line of treatment, and at third-line as monotherapy in patients with $R A S$ wild-type metastatic colorectal cancer [7].

Abnormal EGFR activity is implicated in colorectal cancer pathophysiology as a result of its key role in tumor growth and invasion [2]. However, healthy epithelial cells - predominantly sitting in the skin - also express the EGFR, and so the typical adverse reactions experienced by patients receiving EGFRI therapy manifest in the skin [8-

Future : Medicine 


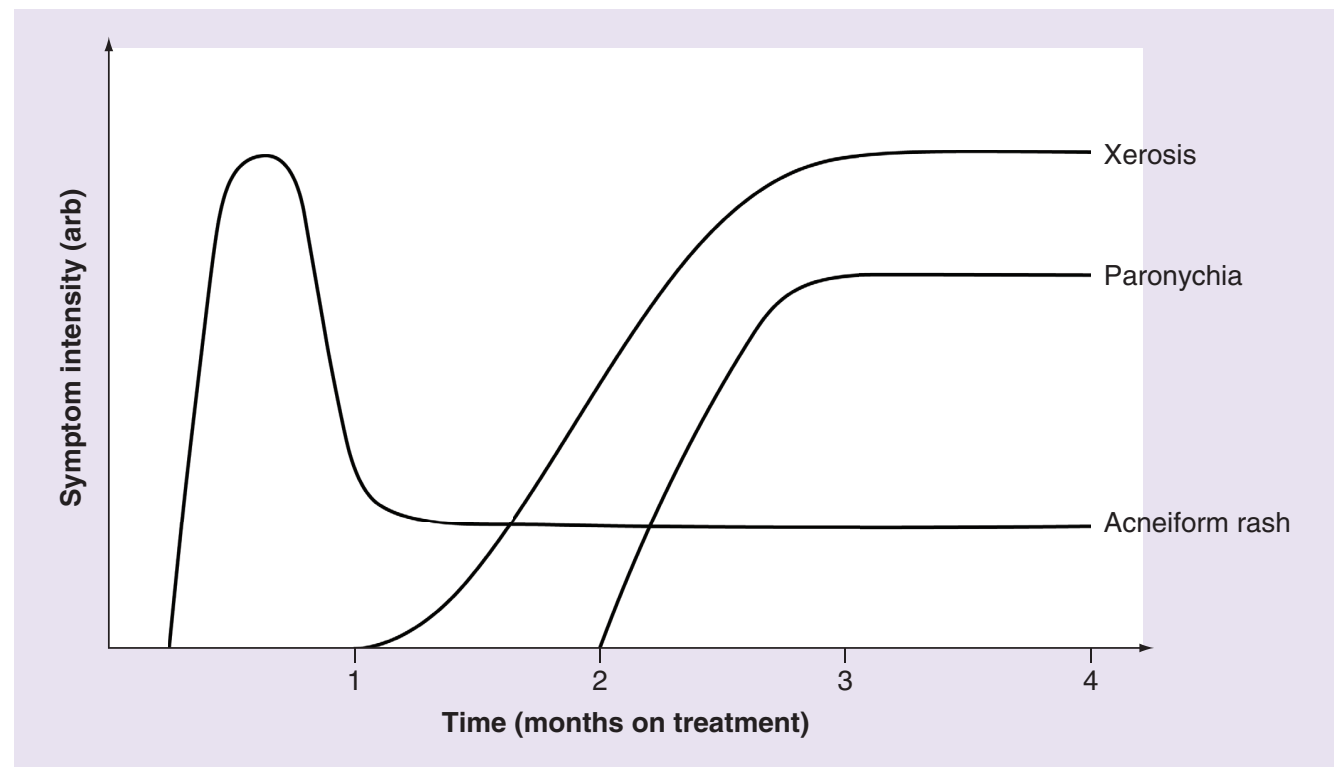

Figure 1. Illustration indicating usual timeline of most common cutaneous toxicities.

10]. Common EGFRI-related adverse events include skin conditions such as xerosis (dry skin), fissures, pruritus, eczema, skin infections and urticaria; nail conditions such as paronychia (suppurative inflammation around the nails); and hair-growth abnormalities, including trichomegaly leading to eye irritation [11]. However, the earliest and most commonly reported cutaneous event is acneiform rash (also known as papulopustular rash), which develops in $60-80 \%$ of patients, usually within the first $1-2$ weeks [8-11]. Figure 1 shows the intensity of the three most common cutaneous toxicities and their emergence over time with ongoing treatment. As adverse-event patterns vary over time, a patient with cutaneous toxicity at 6 months into treatment will likely present with very different symptoms from a patient experiencing toxicity at 2 weeks.

Retrospective studies have shown that the development of cutaneous toxicity and their severity predict EGFRI tumor response and may act as a surrogate marker for treatment efficacy in many tumor types [12-14]. However, cutaneous toxicities - particularly acneiform rash, xerosis and pruritus - can significantly affect a patient's quality of life, to the extent that many physicians feel it necessary to delay or discontinue EGFRI treatment [15], with a potentially negative impact on treatment outcomes, including overall survival $[11,16,17]$. Treatment with EGFRIs has also been shown to cause a substantial economic burden as a result of its associated dermatological adverse effects [18]. Therefore, it is imperative to establish appropriate strategies for the prevention and management of EGFRI-associated dermatological toxicity, especially given that this toxicity does not compromise treatment efficacy.

\section{Optimizing the management of EGFRI-associated cutaneous toxicity}

Chemotherapy-related adverse events, such as nausea, receive close attention and are generally well managed. Given the potential for EGFRI-related cutaneous toxicities to affect quality of life and the continuation of treatment - when relatively simple advice and treatments could limit them - cutaneous toxicities should receive the same level of attention as chemotherapy-related toxicities. There is also a lack of standardization in the care offered to patients receiving EGFRIs to manage cutaneous adverse events within hospitals and between hospitals across Europe. We convened a group of colorectal oncologists, a nurse clinician, oncology pharmacists and a dermatologist with extensive experience of managing cutaneous reactions in patients with colorectal cancer in order to optimize the care that patients receive.

The objective of this review is to provide advice and recommendations on standardising and streamlining the management of patients receiving EGFRIs. The current published evidence was reviewed and combined with clinical experience to propose an algorithm for the timely and effective management of cutaneous toxicities in patients with colorectal cancer receiving EGFRI treatment (Figure 2).

The symptoms that the algorithm focuses on - rash, eczema, xerosis and paronychia - were prioritized on the basis of both their clinical significance and the scope for interventions that could prevent or limit their impact. Hair 


\begin{tabular}{|c|c|c|c|}
\hline Study (year) & Protocol & Factors for consideration & Ref. \\
\hline Lacouture ME et al. (2010) & STEPP & $\begin{array}{l}\text { - Small sample size } \\
\text { - Focus on panitumumab only } \\
\text { - Antibiotic and steroid use in the absence of symptoms }\end{array}$ & [23] \\
\hline MASCC Skin Toxicity Study Group (2011) & $\begin{array}{l}\text { MASCC Skin Toxicity Study Group Clinical } \\
\text { Practice Guidelines }\end{array}$ & $\begin{array}{l}\text { - A broad focus on all EGFRIs and their use in solid tumors } \\
\text { rather than the EGFR monoclonal antibodies used in } \\
\text { colorectal cancer }\end{array}$ & [10] \\
\hline Reguiai Z et al. (2012) & $\begin{array}{l}\text { French interdisciplinary therapeutic } \\
\text { algorithm }\end{array}$ & $\begin{array}{l}\text { - Antibiotic use in the absence of symptoms } \\
\text { - Grading of rash based on percentage of body surface area } \\
\text { affected as per classification used for clinical trials } \\
\text { (NCI-CTCAE) }\end{array}$ & [24] \\
\hline Alberta Health Services' (2015) & $\begin{array}{l}\text { Panitumumab and cetuximab toxicity } \\
\text { management guidelines for patients with } \\
\text { advanced colorectal cancer }\end{array}$ & - Antibiotic and steroid use in the absence of symptoms & [25] \\
\hline
\end{tabular}

and eye symptoms were not included because only basic advice can be given, such as regular trimming of eyelashes and treatment of infections with antibiotics, with the aim of minimizing these symptoms.

\section{Current recommendations \& guidelines}

The management of EGFRI-associated cutaneous toxicities is largely based on personal experience and small scale audits; very few recommendations are evidence based, because of the lack of relevant controlled trials. Several groups have proposed recommendations and reviewed the evidence for managing EGFRI-associated cutaneous toxicities, including 2009 recommendations from a National Comprehensive Cancer Network (NCCN) task force $[10,11,19,20]$. For example, although it was initially proposed that topical vitamin K3 might provide benefit as a prophylactic for cetuximab-induced rash in patients with metastatic colorectal cancer, a recent Phase II, placebo-controlled study of vitamin K3 cream did not support any clinical or immunohistochemical benefit [21]. Similarly, in a recent Phase II, double-blind, vehicle controlled study, vitamin K1 cream as prophylaxis for cetuximab-induced skin toxicity did not reduce the incidence of grade $\geq 2$ skin rash but severity did appear reduced using an alternative and more thorough scale for skin toxicity [22]. There are also several management protocols in existence that provide useful analysis and advice concerning toxicity prophylaxis and management, but these also have limitations (Table 1).

\section{Grading of toxicity}

The grading of adverse cutaneous events is important to validate the benefit of pre-emptive therapy, guide reactive treatment and decide on EGFRI-dose modifications. Such grading often takes into account the level of intervention required to manage the adverse events and the ability to continue treatment. However, grading is often based on the surface area affected, rather than the location of the cutaneous reaction and the subsequent impact on the patient's quality of life. This is true of grading systems such as the National Cancer Institute Common Terminology Criteria for Adverse Events (NCI-CTCAE). An extensive rash in one area, commonly the face, can considerably reduce a patient's quality of life and ability to cope with EGFRI-based chemotherapy. Treating this region appropriately would improve their quality of life to maintain the dose intensity of EGFRI-based chemotherapy and its subsequent efficacy. Further limitations of this system are that it does not cover all the adverse events specific to EGFRI therapy, such as eczema and paronychia, and for the tyrosine kinase EGFRIs at least, has been found to result in underreporting of the incidence of adverse events [26].

There have been concerted efforts to develop more precise and clinically relevant tools to quantify and monitor EGFRI-related skin toxicities, including the MASCC EGFRI Skin Toxicity Tool (MESTT) [27] and the EGFRIrelated Skin Toxicity Index (EGFRISTI) [28]. However, the MESTT requires individual pustules to be counted [27], which is impractical in a busy clinic, and the EGFRISTI is again based on the surface area affected [28]. Tools have also been developed to measure the impact of dermatological symptoms on quality of life such as Skindex-16, a general dermatology tool [29] and the EGFRI-specific Functional Assessment of Cancer Therapy Questionnaire-EGFRI (FACT-EGFRI-18) [30].

Table 2 shows criteria we propose for use with the algorithm to assess the local severity of the cutaneous symptoms. 
Table 2. Proposed grading of severity of EGFRI-associated cutaneous toxicity.

\begin{tabular}{|c|c|c|c|}
\hline \multirow[t]{2}{*}{ Contributing factors } & \multicolumn{3}{|c|}{ Grading } \\
\hline & Mild & Moderate & Severe \\
\hline Impact on patient's quality of life & Not limiting day-to-day life activities & Limiting certain daily activities & Intolerable to patient \\
\hline Intervention needed & Can be self-managed by the patient & Requires several treatments to manage & $\begin{array}{l}\text { Requires intensive local and possibly } \\
\text { systemic treatment to manage }\end{array}$ \\
\hline Ability to continue EGFRI treatment & No dose modification required & No dose modification required & $\begin{array}{l}\text { Treatment discontinuation until } \\
\text { symptoms improve to mild, and possible } \\
\text { dose reduction }\end{array}$ \\
\hline \multirow[t]{5}{*}{ Example appearance } & Skin & & \\
\hline & $\begin{array}{l}\text { Redness and flushing only, with or } \\
\text { without itch }\end{array}$ & $\begin{array}{l}\text { Papules, pustules and irritation } \\
\text { (acneiform) }\end{array}$ & $\begin{array}{l}\text { Crusted, eroded pustular acneiform } \\
\text { lesions } \\
\text { Example of a severe EGRI-induced } \\
\text { acneiform rash: }\end{array}$ \\
\hline & Nails & & \\
\hline & $\begin{array}{l}\text { Nail-fold edema or erythema; } \\
\text { disruption of the cuticle }\end{array}$ & $\begin{array}{l}\text { Edema or erythema with discharge or } \\
\text { nail-plate separation resulting in } \\
\text { discomfort }\end{array}$ & $\begin{array}{l}\text { Edema or erythema with discharge or } \\
\text { nail-plate separation resulting in severe } \\
\text { pain and reduced mobility }\end{array}$ \\
\hline & $\begin{array}{l}\text { Example of mild EGRI-induced } \\
\text { paronychia }\end{array}$ & $\begin{array}{l}\text { Example of moderate EGRI-induced } \\
\text { paronychia }\end{array}$ & $\begin{array}{l}\text { Example of severe EGRI-induced } \\
\text { paronychia }\end{array}$ \\
\hline
\end{tabular}

\section{Recommended management}

The following treatment strategies are proposed for the prevention and management of cutaneous toxicities, as outlined in the algorithm (Figure 2). In summary, these consist of a standard prescription pack and prophylactic advice for all patients starting an EGFRI for the first time (Figure 2A), and a recommendation for the regular review, grading and management of emergent cutaneous adverse events at each cycle from 2 onward (Figure 2B).

These recommendations are based on the available evidence for EGFRI-associated toxicities and our clinical experience and also draw on evidence for the treatment of similar conditions not associated with EGFRI use.

\section{Preventive treatment}

There is evidence that prophylactic action can significantly reduce the incidence of cutaneous toxicities associated with EGFRI therapy compared with reactive management $[23,31,33]$. The STEPP study reported grade 2 or above skin toxicities in $29 \%$ of pre-emptively treated patients compared with $62 \%$ of reactively treated patients (odds ratio: 0.3) [23]. This study used prophylactic oral antibiotics and topical steroids in addition to daily moisturizer and sunscreen from the start of EGFRI treatment. However, the reactive treatment group was only reviewed on a weekly basis meaning it could have been up to 7 days between a symptom emerging and treatment being prescribed. Indeed, the median time from start of treatment to skin toxicity was 2.1 weeks. The J-STEPP study showed that only $50 \%$ of the reactively treated patients had grade 2 or above toxicity (central review at 6 weeks), with onset at a median of 3.2 weeks after the start of treatment [32]. Therefore, we recommend the reactive use of oral tetracyclines and topical steroids promptly once symptoms emerge to avoid unnecessary exposure to the potential side effects of these treatments in patients who are already heavily medicated and who will not ever develop a cutaneous reaction; 
this accounted for $38 \%$ of patients in reactive arm of the STEPP study (where even moisturizer and sunscreen were not used prophylactically), and may be even higher if emollients, antiseptic soap substitutes and sunscreen are used as described here. The onset of grade 2 toxicity is relatively slow (2-3 weeks), so if patients have a supply of antibiotics, emollients and steroid creams available to them, they will have adequate time to start using them before symptoms become severe.

In addition to the medications prescribed for prophylaxis of cutaneous toxicities, advice should be given to patients at EGFRI initiation on self-management and the reasons behind the potential skin reactions. This faceto-face education is vital to maximize adherence and so optimize outcomes. It may also help to prevent medicine wastage. However, it is essential that patients receive consistent advice from all members of their healthcare team.

\section{Sunscreen}

Advice to avoid direct sunlight and to wear sunscreen is common to all guidelines on the initiation of EGFRI therapy because UV light can exacerbate the acneiform rash particularly in patients with fair skin types (Fitzpatrick type 1 and 2) [11]. Additionally, photosensitivity can also be caused by tetracyclines given to suppress inflammation [33], although the association with photosensitivity is less with lymecycline than with other tetracyclines [34].
(A)
Cycle 1

Preventive treatment and advice for all patients commencing EGFRI treatment (cycle 1)

\section{PRESCRIPTION \\ For prophylactic use}

- Light emollient (lotion) applied daily as required

- Antiseptic-containing soap substitute used daily

For reactive use in case of symptoms (redness, dryness, early acneiform spots ${ }^{\dagger}$ )

- Mild topical steroid cream (e.g., $1 \%$ hydrocortisone) +/- antifungal TDS for up to 14 days initially

- Oral tetracycline (e.g., lymecyclineor doxycycline at licensed dose) OD for up to 14 days or, if

\section{GENERAL ADVICE}

- Use sunblock (SPF 50) when outdoors and avoid strong sun and weather extremes

- Avoid hot baths, showers and saunas

- Avoid alcohol-based and fragranced skin-care products that may exacerbate dry skin

- Apply petroleum jelly to periungual skin to create water-proof layer

- Avoid manicure/pedicures and avoid tight fitting shoes

- For patients with pre-existing eczema: intensify usual skin care routine

- For patients with active rosacea, acne or eczema: refer immediately to the dermatology department

Patients should contact the oncology team or pharmacy team at the onset of symptoms and for additional skin-care advice between clinic appointments.

Figure 2. Management of cutaneous toxicities in patients receiving EGFRI. (A) In cycle 1 and (B) from cycle 2 onward.

${ }^{\dagger}$ Acneiform spots include papules, pustules and crusted sores.

¥EGFRI dose interruptions and dose reductions should happen when the skin reactions are intolerable or severe (common terminology criteria for adverse events [CTCAE] grade 3 or above). On the first occurrence, the EGFRI should be interrupted and resumed at the original dose once the adverse event(s) have resolved to grade 2 (mild) or less. On the second and third occurrence, treatment should also be interrupted until symptoms resolve to grade 2 (mild) or less, but should be resumed at a reduced dose of $80 \%$ and $60 \%$, respectively. On the fourth occurrence, treatment should be permanently discontinued.

BD: Twice daily; EGFRI: EGFR inhibitor; OD: Once daily; SPF: Sun protection factor; TDS: Three times daily. 


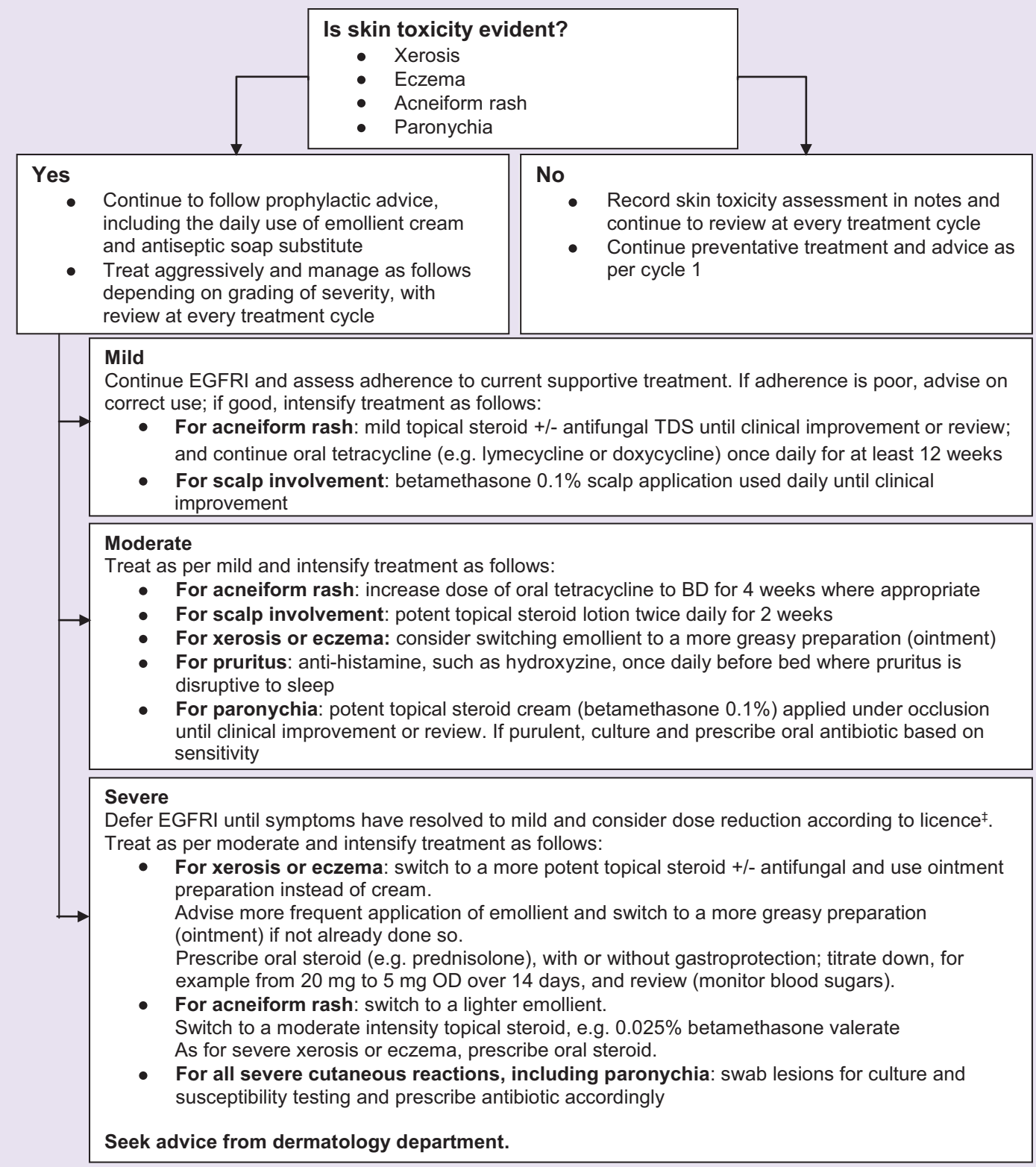

Figure 2. Management of cutaneous toxicities in patients receiving EGFRI (cont.). (A) In cycle 1 and (B) from cycle 2 onward.

${ }^{\dagger}$ Acneiform spots include papules, pustules and crusted sores.

$\ddagger$ EGFRI dose interruptions and dose reductions should happen when the skin reactions are intolerable or severe (common terminology criteria for adverse events [CTCAE] grade 3 or above). On the first occurrence, the EGFRI should be interrupted and resumed at the original dose once the adverse event(s) have resolved to grade 2 (mild) or less. On the second and third occurrence, treatment should also be interrupted until symptoms resolve to grade 2 (mild) or less, but should be resumed at a reduced dose of $80 \%$ and $60 \%$, respectively. On the fourth occurrence, treatment should be permanently discontinued.

BD: Twice daily; EGFRI: EGFR inhibitor; OD: Once daily; SPF: Sun protection factor; TDS: Three times daily. 


\section{Emollients \& soap substitutes}

Conventional soaps and shampoos can dry the skin by removing surface lipids and may irritate the skin as a result of the chemicals they contain [35]. Emollients and soap substitutes create a layer to trap moisture [35] and are recommended pre-emptively and for the duration of EGFRI therapy to maintain hydration. In order to maximize patient adherence, patients should be encouraged to try a variety of emollient products until they find one they have a personal preference for. Damaged skin can be more prone to secondary infection so a soap substitute containing antimicrobial agents, such as Dermol wash lotion, is recommended. This antimicrobial emollient was proven to have a more rapid antibacterial effect than an emollient without antimicrobial and was rated by patients as better than previously used emollients in terms of ease of use and cosmetic acceptability [36]. It should be noted that aqueous cream is not suitable for use as an emollient due to the presence of sodium lauryl sulfate that may cause skin irritation [37].

\section{Antibiotics}

Oral tetracyclines are effective in the treatment of papulopustular acne and rosacea [38]. Aside from the antibiotic properties of tetracyclines, these drugs have an anti-inflammatory effect, acting via the inhibition of matrix metalloproteinases and reducing neutrophil chemotaxis and the production of proinflammatory cytokines. They also have antiangiogenic properties and possibly affect immune modulation via reduced T-cell activation [39].

In the algorithm, we recommend either oral lymecycline or doxycycline, dependent on local availability, once or twice daily at the approved dose. However, lymecycline is preferred as a result of its slightly better adverse event profile, including the lesser risk of photosensitivity, compared with other tetracyclines. For patients in whom tetracyclines are contraindicated, the macrolides, erythromycin or clarithromycin, combine anti-inflammatory and antibiotic effects. Skin infections are most often caused by Staphylococcus while paronychia-associated infections may be caused by Enterococcus and Pseudomonas as well as Staphylococcus; therefore, broad-spectrum antibiotics are useful [11]. However, if skin or nail infections progress, swabs are recommended for culture and sensitivity testing. Controlled trials have shown that antibiotic prophylaxis is associated with a trend toward a reduced severity of rash but no reduction in the overall incidence $[9,40]$. As a result, we recommended that patients are provided with the specified antibiotics to take immediately at symptom onset, but not before.

To help prevent mild paronychia from becoming infected, topical treatment with an antibiotic cream containing mupirocin (active against staphylococcal and streptococcal species found on the skin) is recommended, plus daily soaking of the nails in saline solution.

Topical antibiotics, such as clindamycin gel or metronidazole cream/gel, can also be used for skin rash [41]. However, they often contain preservatives that can irritate the skin so, given the availability of an oral option and in the interests of a streamlined algorithm, topical antibiotics are not recommended.

\section{Antihistamines}

Hydroxyzine is a long-acting and sedating antihistamine that may be useful for patients with sleep disturbances due to pruritus or irritation. However, antihistamines in general do not ameliorate the itch of xerosis or eczema. Care should be taken when prescribing hydroxyzine as there are several contraindications to its use, including caution in elderly patients, as well as interactions with concomitant medications, such as other CNS depressants.

\section{Topical steroids}

Topical steroids are recommended for the treatment of emergent skin reactions from cycle 1 onward. They have anti-inflammatory and antipruritic effects and an accepted role in managing all forms of eczema and other inflammatory skin diseases [41]. The algorithm specifies the short-term use of topical steroids for a maximum of 2 weeks before review to allow antibiotics and other management strategies to take effect, while limiting the risk of rosacea and other steroid-induced adverse events associated with longer-term use. Steroid impregnated occlusive tape (e.g., Haelan Tape ${ }^{\circledR}$ ) is an effective therapeutic dressing for paronychia.

A combined steroid and antifungal formulation is the preferred option for skin reactions at all stages of the algorithm. Histopathological assessment of EGFRI-induced papulopustular rash has recorded the presence of yeast colonies in affected hair follicles (also seen in seborrheic eczema) prompting the use of an antifungal-containing steroid in our protocol [38]. For acneiform rash, we suggest using a steroid cream at cycle 1 and for mild and moderate rash at cycle 2 onward. For xerosis or eczema (moderate or severe at cycle 2 onward), we suggest using an 
ointment because of the higher oil content compared with creams, meaning they are more emollient and effective in delivering the steroid.

\section{Seeking advice from dermatologists}

We recommend that clinicians treating colorectal cancer patients with EGFRIs seek advice from a dermatologist in any case where the patient has significant active skin disease at the start of EGFRI treatment or develops severe symptoms during treatment. A dermatologist will be able to advise on care and suggest more specialist treatments for patients with the most serious skin reactions.

\section{Cosmetics}

The treatments described above can reduce the physical discomfort associated with skin rash, but the visual appearance of the rash can still cause emotional discomfort, particularly if the rash is on the face. Covering the rash with thicker 'camouflage' foundations that are dermatologically tested and hypoallergenic, such as those used by professional makeup artists, can increase a patient's confidence to go out, continue with their daily activities and, therefore, continue EGFRI-based chemotherapy.

\section{Discussion}

Patients with colorectal cancer treated with an EGFRI frequently experience cutaneous toxicities, including rash, eczema, xerosis and paronychia, which can lead to a decreased quality of life, interruption of treatment and a reduction in EGFRI dose. The criteria to assess cutaneous toxicity are not well defined and there is currently a paucity of high quality research in the management of cutaneous toxicity, which has until now led to significant variations in clinical practice.

The algorithm presented has the potential to standardize and optimize the management of EGFRI-induced cutaneous toxicities in patients with colorectal cancer treated with EGFRIs. This algorithm includes the provision of adequate and appropriate prophylaxis and advice to limit the emergence of cutaneous toxicities. The algorithm includes a novel severity grading system that takes into consideration the location and psychological impact of symptoms on the patient rather than simply the surface area affected. The algorithm highlights the importance of patients receiving face-to-face education on skin reactions and their management. An additional and unique benefit of this approach is that it also provides an opportunity to share tips for coping with side effects. For example, patients can be directed toward information on cosmetic foundations that can greatly increase a patient's confidence and quality of life and, therefore, affect their willingness to be compliant with their cancer treatment.

Providing a standardized treatment framework and the implementation of the algorithm presented here will benefit both patients and healthcare professionals ( $\mathrm{HCPs}$ ); it will help all healthcare professionals treating patients with colorectal cancer understand how to manage the cutaneous adverse events arising from EGFRI therapy, including when it is important to refer patients to a dermatologist for specialist management of their symptoms.

An additional benefit of establishing a formal algorithm is that when patients are treated outside of the colorectal cancer department, these other hospital departments are more likely to acknowledge that limiting cutaneous toxicities is essential and that preventative measures should not be stopped unless it is essential. Although terminating these prophylactic and management strategies may not have immediate negative effects, it can give patients the impression that they do not need to continue to follow the advice and can discourage them from being adherent in the long term. Similarly, a formal algorithm is more likely to be respected and closely adhered to by patients than informal advice, which may be seen as optional. Another benefit of the algorithm is that, by encouraging self-management by patients, antibiotics and steroids can be used at the onset of symptoms and not before. The final benefit of the algorithm is the potential to reduce the significant costs related to skin toxicities in patients receiving EGFRI therapy [42]. Implementation of the novel severity grading system and the algorithm presented here have the potential to reduce the emergence of EGFRI-emergent cutaneous toxicities, increase quality of life, increase EGFRI compliance and decrease the direct and indirect impact of the healthcare system.

\section{Future perspective}

Currently, colorectal cancer (even when diagnosed early) is strongly associated with the development of metastatic disease and high mortality rates. The purpose of the algorithm proposed in this article is to help patients and healthcare providers anticipate and prevent and/or manage the cutaneous toxicities associated with EGFRI treatment, which patients find so hard to tolerate. Newer antibodies and tyrosine kinase inhibitors (TKIs) are rapidly 
emerging and subsequently skin rashes are becoming more prevalent. It is likely that these recommendations can be extrapolated and adapted to be used with these newer therapies. It is hoped that appropriate use of this algorithm will improve the tolerance of these therapies, and - in the long-term - patient outcomes.

\section{Executive summary}

\section{Background}

- The availability of the EGFR inhibitors (EGFRIs) cetuximab and panitumumab for the treatment of colorectal cancer has improved survival rates in this patient population.

- These treatments are often associated with cutaneous toxicities - such as xerosis, eczema, acneiform rash, pruritus and paronychia - that can be severe and can influence persistence on therapy.

- Current grading systems for dermatological conditions tend to focus on the size of the affected area rather than the location of the lesions, tend not to be sufficiently specific for cutaneous toxicities associated with EGFRI therapy, and are not always practical for use in a busy clinic. The paucity of high quality research in the management of EGFRI-associated cutaneous toxicity has led to significant variations in clinical practice.

Algorithm for the prevention \& management of EGFRI-associated cutaneous toxicity

- A grading system for the local severity of cutaneous toxicities was developed to take into account the location and psychological impact of symptoms.

- The proposed algorithm includes the provision of a standard prescription pack and prophylactic advice for all patients starting an EGFRI for the first time to encourage self-management.

- It also provides guidance on subsequent review and escalation of treatment to manage symptoms emerging or worsening after cycle 2 of EGFRI therapy.

\section{Conclusion}

- Use of an algorithm such as presented here aims to standardize and optimize the management of EGRFI-associated cutaneous toxicities.

- An increased awareness of adequate prophylaxis and self-management by patients is expected to lead to a reduction in emergent toxicities and related costs together with the maintenance of quality of life and dose intensity.

Financial \& competing interests disclosures

J Beech has been involved with advisory boards or other paid meetings for Merck and Amgen; M Braun has received honoraria from Amgen, Merck, Roche and Servier; MP Saunders has been involved with advisory boards and other paid meetings for Amgen, Merck, Roche, Sanofi, and Servier; J Fenwick is an employee of Merck Serono Ltd., UK (an affiliate of Merck KGaA, Darmstadt, Germany). The authors have no other relevant affiliations or financial involvement with any organization or entity with a financial interest in or financial conflict with the subject matter or materials discussed in the manuscript apart from those disclosed.

Editorial support was provided by Succinct Medical Communications and funded by Merck Serono Ltd.

\section{Open access}

This work is licensed under the Attribution-NonCommercial-NoDerivatives 4.0 Unported License. To view a copy of this license, visit http://creativecommons.org/licenses/by-nc-nd/4.0/

\section{References}

1. Ferlay J, Soerjomataram I, Dikshit R et al. Cancer incidence and mortality worldwide: sources, methods and major patterns in GLOBOCAN 2012. Int. J. Cancer 136(5), e359-e386 (2015).

2. Moriarity A, O’Sullivan J, Kennedy J, Mehigan B, McCormick P. Current targeted therapies in the treatment of advanced colorectal cancer: a review. Ther. Adv. Med. Oncol. 8(4), 276-293 (2016).

3. Rafiemanesh H, Mohammadian-Hafshejani A, Ghoncheh $\mathrm{M}$ et al. Incidence and mortality of colorectal cancer and relationships with the human development index across the world. Asian Pac. J. Cancer Prev. 17(5), 2465-2473 (2016).

4. Hohla F, Winder T, Greil R, Rick FG, Block NL, Schally AV. Targeted therapy in advanced metastatic colorectal cancer: current concepts and perspectives. World J. Gastroenterol. 20(20), 6102-6112 (2014).

5. Kohne C, Bokemeyer C, Heeger S, Sartorius U, Rougier P, Cutsem EV. Efficacy of chemotherapy plus cetuximab according to metastatic site in KRAS wild-type metastatic colorectal cancer (mCRC): analysis of CRYSTAL and OPUS studies . J. Clin. Oncol. 29(Suppl.), Abstract 3576 (2011).

6. Lacouture ME. Mechanisms of cutaneous toxicities to EGFR inhibitors. Nat. Rev. Cancer 6(10), 803-812 (2006). 
7. Cutsem VE, Cervantes A, Adam R et al. ESMO consensus guidelines for the management of patients with metastatic colorectal cancer. Ann. Oncol. 27(8), 1386-1422 (2016).

8. Lynch TJ, Kim ES, Eaby B, Garey J, West DP, Lacouture ME. Epidermal growth factor receptor inhibitor-associated cutaneous toxicities: an evolving paradigm in clinical management. Oncologist 12(5), 610-621 (2007).

9. Jatoi A, Rowland K, Sloan JA et al. Tetracycline to prevent epidermal growth factor receptor inhibitor-induced skin rashes: results of a placebo-controlled trial from the North Central Cancer Treatment Group (N03CB). Cancer 113(4), 847-853 (2008).

10. Lacouture ME Anadkat MJ Bensadoun RJ et al. Clinical practice guidelines for the prevention and treatment of EGFR inhibitor-associated dermatologic toxicities. Support. Care Cancer 19(8), 1079-1095 (2011).

11. Burtness B, Anadkat M, Basti S et al. NCCN Task Force report: management of dermatologic and other toxicities associated with EGFR inhibition in patients with cancer. J. Natl Compr. Cancer Netw. 7 (Suppl. 1), S5-S21; quiz S22-S24 (2009).

12. LoRusso P. Toward evidence-based management of the dermatologic effects of EGFR inhibitors. Oncol. Williston Park N. 23(2), 186-194 (2009).

13. Saltz LB, Meropol JN, Loehrer JP, Needle MN, Kopit J, Mayer JR. Phase trial II of cetuximab in patients with refractory colorectal cancer that expresses the epidermal growth factor receptor. J. Clin. Oncol. 22(7), 1201-1208 (2004).

14. Cunningham D, Humblet $Y$, Siena $S$ et al. Cetuximab monotherapy and cetuximab plus irinotecan in irinotecan-refractory metastatic colorectal cancer. N. Engl. J. Med. 351(4), 337-345 (2004).

15. Paul T, Schumann C, Rüdiger $S$ et al. Cytokine regulation by epidermal growth factor receptor inhibitors and epidermal growth factor receptor inhibitor associated skin toxicity in cancer patients. Eur. J. Cancer 50(11), 1855-1863 (2014).

16. Boone SL, Rademaker A, Liu D, Pfeiffer C, Mauro JD, Lacouture ME. Impact and management of skin toxicity associated with anti-epidermal growth factor receptor therapy: survey results. Oncology 72(3-4), 152-159 (2007).

17. Segaert S, Cutsem VE. Clinical management of EGFRI dermatologic toxicities: the European perspective. Oncol. Williston Park N. 21(11 Suppl. 5), 22-26 (2007).

18. Ray S, Bonthapally V, Holen KD et al. Economic burden of dermatologic adverse drug reactions in the treatment of colorectal, non-small cell lung, and head and neck cancers with epidermal growth factor receptor inhibitors. J. Med. Econ. 16(2), 221-230 (2013)

19. Segaert $\mathrm{S}$, Tabernero J, Chosidow $\mathrm{O}$ et al. The management of skin reactions in cancer patients receiving epidermal growth factor receptor targeted therapies. J. Dtsch Dermatol. Ges. J. Ger. Soc. Dermatol. 3(8), 599-606 (2005).

20. Hofheinz R-D, Deplanque G, Komatsu Y et al. Recommendations for the prophylactic management of skin reactions induced by epidermal growth factor receptor inhibitors in patients with solid tumors. Oncologist 21(12), 1483-1491 (2016).

21. Eriksen JG, Kaalund I, Clemmensen O, Overgaard J, Pfeiffer P. Placebo-controlled Phase study II of vitamin K3 cream for the treatment of cetuximab-induced rash. Support. Care Cancer 25(7), 2179-2185 (2017).

22. Hofheinz R-D, Lorenzen S, Trojan J et al. EVITA - a double-blind, vehicle-controlled, randomized Phase trial II of vitamin K1 cream as prophylaxis for cetuximab-induced skin toxicity. Ann. Oncol. 29(4), 1010-1015 (2018).

23. Lacouture ME, Mitchell EP, Piperdi B et al. Skin toxicity evaluation protocol with panitumumab (STEPP), a Phase II, open-label, randomized trial evaluating the impact of a pre-emptive skin treatment regimen on skin toxicities and quality of life in patients with metastatic colorectal cancer. J. Clin. Oncol. 28(8), 1351-1357 (2010).

24. Reguiai Z, Bachet JB, Bachmeyer C et al. Management of cutaneous adverse events induced by anti-EGFR (epidermal growth factor receptor): a French interdisciplinary therapeutic algorithm. Support. Care Cancer 20(7), 1395-1404 (2012).

25. Alberta Health Services. Panitumumab and Cetuximab Toxicity Management Guidelines (2015). www.albertahealthservices.ca/assets/info/hp/cancer/if-hp-cancer-guide-panitumumab.pdf

26. Chan A, Tan EH. How well does the MESTT correlate with CTCAE scale for the grading of dermatological toxicities associated with oral tyrosine kinase inhibitors? Support. Care Cancer 19(10), 1667-1674 (2011).

27. MASCC. EGFR Inhibitor Skin Toxicity Tool (MESTT). www.mascc.org/MESTT

28. Lisi P, Bellini V, Bianchi L. The epidermal growth factor receptor inhibitor-related Skin Toxicity Index (EGFRISTI): a new tool for grading and managing skin adverse reactions to epidermal growth factor receptor inhibitors. Oncology 87(5), 311-319 (2014).

29. Chren MM, Lasek JR, Sahay AP, Sands LP. Measurement properties of Skindex-16: a brief quality-of-life measure for patients with skin diseases. J. Cutan. Med. Surg. 5(2), 105-110 (2001).

30. Wagner LI, Berg SR, Gandhi M et al. The development of a Functional Assessment of Cancer Therapy (FACT) questionnaire to assess dermatologic symptoms associated with epidermal growth factor receptor inhibitors (FACT-EGFRI-18). Support. Care Cancer 21(4), 1033-1041 (2013).

31. Sinclair R. Anticipating and managing the cutaneous side effects of epidermal growth factor receptor inhibitors. Asia Pac. J. Clin. Oncol. 10(Suppl. 1), 11-17 (2014).

32. Kobayashi Y, Komatsu Y, Yuki S et al. Randomized controlled trial on the skin toxicity of panitumumab in Japanese patients with metastatic colorectal cancer: HGCSG1001 study; J-STEPP. Future Oncol. 11(4), 617-627 (2015). 
33. Bjellerup M, Ljunggren B. Differences in phototoxic potency should be considered when tetracyclines are prescribed during summer-time. A study on doxycycline and lymecycline in human volunteers, using an objective method for recording erythema. $B r$. J. Dermatol. 130(3), 356-360 (1994).

34. Lymecycline 408mg capsules, hard - Summary of Product Characteristics (SPC) - (eMC). www.medicines.org.uk/emc/medicine/26940

35. Holden C, English J, Hoare C et al. Advised best practice for the use of emollients in eczema and other dry skin conditions. J. Dermatol. Treat. 13(3), 103-106 (2002).

36. Whitefield DM. Effectiveness of a new antimicrobial emollient in the management of eczema/dermatitis. J. Dermatol. Treat. 9(2), 103-109 (1998).

37. Aqueous cream: contains sodium lauryl sulfate which may cause skin reactions, particularly in children with eczema. www.mhra.gov.uk/safety-public-assessment-reports/CON251956

38. Griffiths C, Barker J, Bleiker T, Chalmers R, Creamer D. Rook's Textbook of Dermatology (9th Edition). Wiley (2016).

39. Sapadin AN, Fleischmajer R. Tetracyclines: nonantibiotic properties and their clinical implications. J. Am. Acad. Dermatol. 54(2), 258-265 (2006).

40. Scope A, Agero AL, Dusza SW et al. Randomized double-blind trial of prophylactic oral minocycline and topical tazarotene for cetuximab-associated acne-like eruption. J. Clin. Oncol. 25(34), 5390-5396 (2007).

41. Yarbrough KB, Neuhaus JK, Simpson EL. The effects of treatment on itch in atopic dermatitis. Dermatol. Ther. 26(2), 110-119 (2013).

42. Eilers RE Jr, Gandhi M, Patel JD et al. Dermatologic infections in cancer patients treated with epidermal growth factor receptor inhibitor therapy. J. Natl Cancer Inst. 102(1), 47-53 (2010). 
